\title{
Explaining environmental health behaviors: evidence from rural India on the influence of discount rates
}

\author{
STIBNIATI S. ATMADJA
}

Center for International Forestry Research (CIFOR) - Ethiopia, c/o ILRI Campus, PO Box 5689, Addis Ababa, Ethiopia.

Email:s.atmadja@cgiar.org

ERIN O. SILLS

North Carolina State University, USA.

Email: sills@ncsu.edu

SUBHRENDU K. PATTANAYAK

Duke University, USA.

Email: subhrendu.pattanayak@duke.edu

JUI-CHEN YANG

Pacific Economic Research, LLC, USA.

Email: yangjuichen@gmail.com

\section{SUMEET PATIL}

Network for Engineering and Economics Research and Management (NEERMAN), India.

Email: srpatil@neerman.org

Submitted 7 July 2014; revised 29 January 2016, 21 September 2016; accepted 22 November 2016; first published online 7 March 2017

\footnotetext{
ABSTRACT. The authors examine whether high personal discount rates help explain why and which households in developing countries under-invest in seemingly low-cost options to avert environmental health threats, including bednets, clean cooking fuels, individual household latrines, water treatment and handwashing. First, the authors elicit personal discount rates by combining a simple randomized experiment with detailed surveys of over 10,000 rural households in Maharashtra, India. Personal discount rates

The authors acknowledge RTI International and the World Bank for financial support, and Tom Holmes, Mitch Renkow, Eswaran Somanathan, two anonymous referees and conference participants at EAERE and the Pacific Conference for Development Economics for their helpful comments.
} 
are lower for women, for better-off households, and for households who can access formal credit. Secondly, they show that the discount rate is negatively related to a suite of behaviors that mitigate environmental health threats, from very low-cost steps like washing hands to more significant investments like household latrines, even after controlling for socio-economic status, access to credit, public infrastructure and services, and relevant beliefs.

\section{Introduction}

Developing countries face myriad environmental concerns, from water and air quality to vector-borne diseases. Among low-income countries, respiratory infections, diarrheal diseases and malaria are among the top 10 causes of death. For example, household air pollution is now the second leading risk factor in the global burden of disease, and in India alone, almost 385,000 children under five years of age die each year from diarrheal diseases related to poor water quality and sanitation (UNICEF, 2009). These diseases impose significant economic burdens on households in terms of both reduced productivity and medical expenditures. Additionally, these environmental stressors and the resulting infections can lead to deferred but potentially sizeable 'epigenetic' penalties: exposures during the fetal and early stages of life lead to height loss, chronic health harms and damages to cognitive and non-cognitive skills, all of which can transmit across generations (Dasgupta, 2013).

While improved infrastructure (e.g., piped water) is clearly critical, individual households can also mitigate these environmental health threats by engaging in 'environmental health behaviors' such as washing hands, treating drinking water, using latrines, switching to cleaner fuels and sleeping under mosquito bednets (Pattanayak and Pfaff, 2009). We refer to averting behaviors related to environmental health as 'environmental health behaviors' throughout the text, following Pattanayak and Pfaff (2009), who refer to bednets, taps, toilets and cookstoves as environmental health technologies, and the associated behavioral choices as environmental health behaviors. Other literature refers to these as health-seeking, preventive, coping or averting behaviors. These actions are not costless, but they appear to require relatively low investments today in comparison to the diseases that they help prevent in the future.

Households in developing countries, however, engage in these environmental health behaviors at surprisingly low rates (Bruce et al., 2006; Cairncross and Valdmani, 2006; Luby et al., 2008, 2009; Pattanayak and Pfaff, 2009; Lewis and Pattanayak, 2012; Wendland et al., 2014). This seemingly myopic behavior has been considered a contributing factor in the vicious cycle of poverty traps in the rural areas of many developing countries (Dasgupta, 2013). One possible reason is that households heavily discount future environmental health benefits: that is, the present value of these benefits is not high enough to offset the present costs (Pant et al., 2014). This is consistent with the assertion - and some empirical evidence that personal discount rates are high in developing countries (WCED, 1987; Cardenas and Carpenter, 2008).

Previous literature has examined how discount rates are linked to both addictive ('hot') behaviors like smoking or drug use (Chapman, 
2005), and ('cool') behaviors whose benefits manifest only after many years, such as healthy diet and exercise (Hall and Fong, 2007). The discount rate is the conventional measure of how individuals weigh the utility of outcomes that occur at different times. It reflects time preference or impatience (preference for current over future utility), expected changes in the marginal utility of consumption, access to credit, and alternative investment opportunities (Pender, 1996). However, it is not observable and presents a challenge for measurement. The most common approach has been to pose either hypothetical or incentivized choices between monetary payments at different points in time. This approach has been applied frequently in the lab, especially with students in developed countries, and less frequently in the field (e.g., Harrison et al., 2002; Eckel et al., 2004). Carpenter et al. (2005) discuss the benefits and challenges of posing questions in the field. For example, field experiments that offer payments in future years clearly depend on the credibility of the research team, which may vary across respondents (Cardenas and Carpenter, 2008). Partly for this reason, many field applications have involved hypothetical time tradeoff questions posed in household surveys. A small but increasing number of these are in developing countries. Examples include: Poulos and Whittington (2000), who asked about lives saved now vs. 2-10 years into the future; Kirby et al. (2002), who posed the question in terms of candies now vs. 7-157 days later; Anderson et al. (2004), who asked about debt repayment 1 day-1 year from now; and Botelho et al. (2006), who asked about smaller monetary payments now vs. larger monetary payments in 1-25 months.

In the next section of this paper, we review the literature to reconfirm the knowledge gap and present a simple conceptual framework. In section 2, we explain our strategy for estimating discount rates from a randomized choice experiment with approximately 10,000 households from approximately 250 villages in Maharashtra, India (Pattanayak et al., 2010). The choice experiment presented randomized time tradeoff questions to households as part of a survey that also elicited information on socio-economic and demographic characteristics, and environmental health behaviors. This allows us to model responses to the time tradeoff questions as a function of factors expected to influence the personal discount rate: credit access, socio-economic status, and intrinsic biological characteristics that have been found related to time preferences, i.e., sex and age. We then model the relationships between environmental health behaviors and the estimated personal discount rate, controlling for relevant covariates. In the online appendix, available at http:/ / doi.org/10.1017/S1355770X17000018, we show that the estimation results for environmental health behaviors are qualitatively similar if we model them as a function of an estimated rate of time preference, that is, if we include only sex and age in the first stage of the model. In the concluding section, we briefly summarize and discuss some policy implications.

\subsection{Literature review}

The rate of time preference describes the rate at which the utility of a good dissipates solely from delaying consumption, or impatience. Time preferences 
have been shown to vary with age and sex (e.g., Rogers, 1994; Anderson et al., 2004; Rubalcava et al., 2009; Tanaka et al., 2010). However, the rate at which individuals discount future outcomes also depends on their access to credit, expected changes in the marginal utility of consumption, and the opportunity cost of alternative investments. Future outcomes are 'discounted', i.e., have less value, compared to the same outcomes enjoyed now (Samuelson, 1937). Hence, individuals who have lower discount rates should be more likely to invest in future-oriented outcomes, such as environmental health behaviors. The personal discount rate has been tested as a determinant of a range of behaviors involving intertemporal tradeoffs such as savings (Ashraf et al., 2006), debt (Meier and Sprenger, 2010), credit scores (Arya et al., 2013), gambling (Dixon et al., 2003), crime (Nagin and Pogarsky, 2004), investment in children (Agee and Crocker, 2000), and participation in unhealthy behaviors (Lawless et al., 2013; Story et al., 2014). This includes a small but growing number of studies in developing countries (Lumley, 1997; Godoy et al., 1998; Nielsen, 2001; Yesuf, 2004; Bauer and Chytilová, 2010; Bauer et al., 2012; Liebenehm and Waibel, 2014; Rieger, 2015; Nguyen et al., 2016).

Chabris et al. (2008) found that, in comparison to the other variables used in their study, the discount rate has the most explanatory power for modelling behaviors that involve intertemporal tradeoffs. However, this still needs field testing with samples drawn from different countries and cultures (Henrich et al., 2010). In particular, there are differences between developed and developing countries. Discount rates tend to be higher in developing countries (Poulos and Whittington, 2000), and characteristics such as sex and age have different explanatory power (Anderson and Gugerty, 2009). The key public health issues also differ, with the populations of developing countries being much more affected by environmental health concerns related to sanitation, drinking water, indoor air quality and vector-borne diseases.

Many studies in developed countries have sought to understand how addictive and risky behaviors are related to discount rates. In contrast to environmental health behaviors, these unhealthy behaviors have present benefits with future costs (e.g., Hall and Fong, 2007). High discount rates have often been found to be positively related with unhealthy behaviors such as substance abuse (Vuchinich and Simpson, 1998; Kirby and Petry, 2004), risky sexual behavior (Chesson et al., 2006), and smoking (Fong and Hall, 2003; Khwaja et al., 2006; Scharff and Viscusi, 2011). In comparison, relatively fewer studies have empirically confirmed relationships between discount rates and preventive behaviors such as cancer screening tests (Orbell et al., 2004; Picone et al., 2004; Levy et al., 2006; Bradford, 2010). Chapman (2005) proposes that personal discount rates are related to addictive behaviors (e.g., smoking) but not to disease prevention behaviors (e.g., vaccination). This domain specificity in the influence of discount rates could be related to the relative temptation of different domains (e.g., consumption of alcohol) to the individual (Tsukayama and Duckworth, 2010). While most studies focus on adults, discount rates have also been found to be related to addictive behaviors and savings among adolescents (Sutter et al., 2013). 


\subsection{Conceptual framework}

To model environmental health behaviors, consider a person who maximizes the utility from health $(H)$ and a bundle of other goods and services $(X)$ constrained by full income, in the current $\left(X_{0}\right)$ and future $\left(X_{1}\right)$ periods. Health in the present $\left(H_{0}\right)$ and a set of personal-, household- and community-level characteristics $\left(Z_{y}\right)$, including local environmental quality, are given. Health in the future $\left(H_{1}\right)$ is a function of those characteristics and public services $\left(Z_{y}\right)$ and preventive actions $X_{H, 0}$ taken in the present at price $p_{H}$. Investing in environmental health reduces current utility $\left(V_{0}\right)$ by reducing consumption of other goods $X_{0}$, but increases future utility $\left(V_{1}\right)$ by increasing future health $\left(H_{1}\right)$. The present value of future utility $\left(V_{1}\right)$ is discounted by a factor $(\delta)$ that is a function of the personal discount rate $(\rho)$, which in turn is determined by intrinsic characteristics such as sex and age, and economic conditions such as socio-economic status and access to credit $\left(Z_{\rho}\right)$. The most common functional form for $\delta$ is constant exponential discounting: $\delta=e^{-\rho . t}$, where $\rho$ is a constant discount rate. The utility function in both periods is:

$$
\begin{aligned}
V= & V_{0}+\delta V_{1}=V_{0}\left(X_{0}-p_{H} X_{H, 0}, H_{0}, Z_{y}\right) \\
& +\delta\left(\rho\left(Z_{\rho}\right)\right) \cdot V_{1}\left(X_{1}, H_{1}\left(X_{H, 0}\right), Z_{y}\right)
\end{aligned}
$$

and the first-order conditions for utility maximization yield

$$
0=-p_{H} \frac{\partial V_{0}}{\partial X_{H, 0}}+\delta \cdot \frac{\partial V_{1}}{\partial H_{1}} \cdot \frac{\partial H_{1}}{\partial X_{H, 0}} .
$$

First-order conditions (2) show that households set the marginal value of reduced consumption in the current period equal to the discounted marginal value of increased health in the future. Decisions about environmental health behaviors therefore depend on their current cost, their marginal productivity in terms of future health, the marginal utilities of consumption of goods and services in the present vs. health in the future, and personal discount rates. All of these factors are influenced by exogenous characteristics, $Z_{y}$. At the time decisions about behaviors are made, the future utility function is unknown. Thus, it is really expectations about the future marginal utility of health and marginal productivity of the behavior that matter. This suggests that choices about investing in environmental health behaviors are likely to depend on beliefs about disease prevention, access to information, the income constraint and public services (which either complement or substitute for private environmental health behaviors), as well as personal discount rates.

While the notion of discount rates that are stable across different time periods and different goods has been contested (e.g., Meerding et al., 2010), it is implicit in most economic analysis (Frederick et al., 2002). In this paper, we maintain the assumption of constant exponential rate discounting as a straightforward way to summarize the factors driving responses to monetary tradeoff questions. Since personal discount rates $(\rho)$ are inversely related with the discounting function $\delta$, individuals with higher discount rates are expected to invest less in goods related to their future welfare, 
such as environmental health behaviors, compared to those with lower discount rates. People may have higher discount rates because they cannot borrow to finance investments in the future, because they expect to have higher income and therefore lower marginal utility of consumption in the future, because they have good alternative investment opportunities, or simply because they place higher value on current utility relative to future utility (i.e., they are impatient). All of these factors are reflected in the personal discount rate $(\rho)$.

The literature suggests that personal discount rates vary as a function of factors $\left(Z_{\rho}\right)$ including demographics, education, economic status and access to credit. Factors that influence time preferences (or impatience) include age and sex (e.g., Anderson et al., 2004; Rubalcava et al., 2009; Bauer and Chytilová, 2010; Tanaka et al., 2010), and education (Holden et al., 1998; Kirby et al., 2002; Botelho et al., 2006). The theory of endogenous time preferences (Becker and Mulligan, 1997) suggests, and Kirby et al. (2002) confirm, a nonlinear relationship between age and discount rates. Personal discount rates are also influenced by socio-economic status and credit access, partly because of associated differences in the marginal utility of consumption and opportunity cost of time (Pender, 1996; Poulos and Whittington, 2000).

While we conceptualize responses to the time tradeoff questions as a function of personal discount rates, as a robustness check we also model those responses as a function of just the subset of intrinsic personal characteristics that have been found to be related to time preferences, specifically sex and age. We then compare estimation results for models of environmental health behaviors as a function of personal discount rates and as a function of personal time preferences, controlling for the same set of covariates.

\section{Methods}

\subsection{Data}

The data are from a study of water and sanitation programs, including environmental health behaviors, by the World Bank and Research Triangle Institute in rural Maharashtra, India (World Bank, 2008). A key part of the study was a large 2005 survey of over 10,000 households from approximately 250 villages in four districts of Maharashtra, representing a range of eco-climatic zones. The villages were sampled from districts where the state government, with financial support (soft loan) from the World Bank, planned to implement water and sanitation programs. Approximately 50 households were sampled from each study village. Because of concern about environmental health outcomes for children, the survey screened for and included only households with at least one child under the age of five and interviewed only adults familiar with the care and wellbeing of the children in the household. Thus, the sample was not intended to be representative of the population. In comparison to the general population, our respondents are predominantly primary caregivers of the young children, i.e., mostly women of childbearing age. Nonetheless, our sample of households is typical of the rural Indian population, with large families, many 
young children, and low rates of education and literacy. ${ }^{1}$ Further details of the study and sampling protocol can be found in Pattanayak et al. (2009).

The survey questionnaire drew on the Living Standards Measurement Surveys, modified by study investigators for the rural Indian context over the course of several related studies and developed through extensive focus groups and pre-testing. The survey was implemented by a team from TNS International (an international survey firm), with supervision by study investigators. TNS field staff were not involved in any of the government's programmatic activities or in any of the scientific analysis plans or reporting. Thus, any measurement errors in data collection should be classical and not related to the outcomes studied in this paper. The survey questions focused on sociodemographic, economic and environmental health behaviors, including the dependent variables (i.e., the environmental health behaviors) used in our analysis. The module that elicited personal discount rates is described in detail in section 2.2.

Summary statistics for variables representing the determinants of the personal discount rate, $Z_{\rho}$, as well as their expected correlation with the discount rate are presented in table A1 of the online appendix. As suggested by the conceptual framework, we contend that 'purer' biological variables such as age and sex are correlated with time preferences, whereas socio-economic status and credit are also correlated with discount rates. We measure socio-economic status by the percentage of household budget spent on staple food and the first principal component of the quality of housing (materials and number of rooms), presence of an electricity connection, indicator for whether they own the land, and the weighted sum of livestock (cf., Filmer and Pritchett, 2001; Jalan et al., 2009). Credit access is characterized by the response to a survey question: can the respondent borrow Rs. 5,000 (approximately US\$100) from formal lending institutions? Over 70 per cent of respondents say that they can do their own household accounts, reflecting a high level of numeracy that is likely to have helped them understand the time tradeoff questions, and that may itself be inversely related to discount rates.

Summary statistics for the environmental health behaviors being estimated $\left(X_{H, 0}\right)$ and their determinants $\left(Z_{H}\right)$ are presented in online appendix table A2 Determinants include: (i) education and literacy, which affect access to and understanding of health information; (ii) socio-economic status and credit access; and (iii) current economic condition, represented

${ }^{1}$ In 2005-2006, the National Family Health Survey (NFHS-3) interviewed a representative sample of urban and rural households in Maharashtra. Mean values for our sample are similar to mean values for the NFHS rural sample (in parentheses): age of household head, 49 (45); family size, 7.3 (6.1); number of children under five, 1.6 (1.7); mother's age, 25 (25); percentage of mothers with no education, 34 (32); percentage of mothers with primary education, 14 (12); percentage of households with private taps, 28 (36); percentage with private toilets, 13, (24); percentage with bicycles, 35 (36); percentage with telephones, 16 (13); and percentage with water pumps, 9.3 (10.7). The differences between our sample and the NFHS sample with regard to specific infrastructure are likely to be because the project we drew from targeted communities without piped water and sanitation. 
by total monthly expenditures and whether or not they have suffered a recent crisis, which affects the affordability of environmental health behaviors. In addition, we control for concern about disease in the village and access to public infrastructure and services, as measured by minutes required to walk from home to health clinic and to village center. Because the household survey focused on water supply, we have additional covariates describing household access to water and perceptions regarding waterborne diseases, such as whether diarrhea is preventable.

To assess whether personal discount rates influence household actions to prevent the most common environmental health problems (water-borne and washed diseases, respiratory tract infections and malaria), we estimate five behavioral models of the probability that households: (i) treat their water; (ii) wash hands before eating and after defecation; (iii) have a private household latrine; (iv) cook with clean fuels such as LPG; and (v) have a bednet. Diarrhea is mainly spread through fecal-oral contact, and thus the first three behaviors are key to its prevention (Fewtrell et al., 2005; Cairncross and Valdmani, 2006). In our study area, water treatment methods include boiling, straining, mixing in coagulants (e.g., alum or potash tablets that coagulate solid particles to decrease turbidity) or disinfectants (bleach/chlorine), or a combination of these methods. Most respondents report washing their hands either before eating or after defecation, but only 44 per cent wash at both times. About 12 per cent of households have and use their own pit latrines, with the rest engaging in open defecation. Globally, around 4.3 million deaths are attributed to household air pollution, primarily associated with emissions from traditional (solid biomass) cookstoves. Only 11 per cent of surveyed households use LPG or biogas ('clean fuels'). Malaria is another major cause of mortality and morbidity in India, with 24 million cases per year according to the WHO. There has been substantial progress in combatting malaria around the globe, largely due to campaigns to promote household use of bednets. Among surveyed households, 21 per cent report that they own and use a bednet.

\subsection{Discount rate elicitation}

Following the 'choice task method' (Cardenas and Carpenter, 2008), respondents were asked a series of five hypothetical questions about their preferred timing for a gift of money, or 'payment'. The question was introduced as follows: 'We are trying to understand how individuals in this village make decisions when faced with a choice between an outcome in the near future compared to an outcome in the distant future. Suppose that someone offers you a gift of some money.' In each question, there are three possible answers: (a) smaller sooner (SS), (b) larger later (LL), or (c) Uncertain. For example:

If you select option A, you will receive a payment of Rs. 1,000 now. If you select Option B, then you will receive Rs. 2,000 after 12 months. Which option would you choose?

\section{[1] Option A [2] Option B [3] Uncertain}

There were four versions of the time tradeoff questions to which respondents were randomly assigned. Each version had five questions with 
different combinations of payments at different times. The implied personal discount rates (i.e., discount rates that would make the respondent indifferent between the options $\mathrm{A}$ and $\mathrm{B}$ ) range from 0.8 to 10.0 per cent per month. The specific amounts and associated implied discount rates were developed through pre-testing with 250 households. The larger payments offered are significantly larger than typical monthly income. Hence, these are not trivial sums of money.

The SS options include immediate payments and payments in three months. The LL options include payments in five, 12 or 15 months. Through the series of five questions, we derive the upper and lower bounds of each respondent's personal discount rate (i.e., $\rho_{\text {High }}$ and $\left.\rho_{\text {Low }}\right)$. $\rho_{\text {High }}$ are missing for respondents who always choose SS, while $\rho_{\text {Low }}$ are missing for those who always choose LL. This approach to elicitation of discount rates has been frequently employed (Frederick et al., 2002). Some respondents gave inconsistent responses, i.e., sometimes choosing responses that indicated a lower personal discount rate and sometimes choosing responses that indicated a higher discount rate. Following the literature (e.g., Pender, 1996; Bauer and Chytilová, 2009), we eliminated these inconsistent responses from the analysis. There were no statistically significant differences in means between the 8,297 respondents with consistent and the 1,908 respondents with inconsistent or missing responses across the variables used to model personal discount rates, except that a higher percentage of consistent respondents could do household accounts (i.e., there was a higher rate of numeracy among consistent respondents; see online appendix table A3).

Our sample and methods are similar to other studies of discount rates in India. Specifically, Pender (1996) estimated the discount rate of rural households in the state of Andhra Pradesh, using a small sample survey to elicit preferences over different SS vs. LL real rewards. He found the median annual discount rate to be higher than 50 per cent. Using a similar approach, Bauer et al. (2012) estimated a mean three-month discount rate of 21.6 per cent, based on a survey of 573 rural households in the state of Karnataka. Other studies have employed different methods to estimate the discount rates of the national or urban Indian population. Based on a review of the literature, Ekholm et al. (2010) extrapolated a discount rate of 62-74 per cent per year for households in rural India. Shanmugam (2006) used methods developed by Moore and Viscusi (1988) to estimate discount rates based on individual choices of jobs with different levels of risk. He estimated a discount rate of 7.6 per cent based on the observed occupational choices of 522 blue-collar workers in Madras. In comparison to these studies, we have a much larger sample, because our data were collected as part of a larger study on sanitation and water. That also precluded the use of real incentives, because of the difficulty and cost of establishing a credible system for delivering payments to thousands of rural households over a number of months. While willingness to pay estimates derived from hypothetical choices have found larger than actual payments across a wide range of environmental goods, there are fewer discrepancies in experiments involving private goods, non-students and choice-based elicitation mechanisms such as are used in this study (List and Gallet, 2001; 
Murphy et al., 2003). In the domain of temporal choices, Johnson and Bickel (2002) found consistency in responses to time tradeoff (delay discounting) questions with hypothetical vs. real payments across a range of payment magnitudes.

Another aspect of our elicitation method is that the hypothetical payments are 'found money' rather than endowments (i.e., owned by respondents prior to the survey). Individuals may have more aversion to losing access to things they already have, compared to gaining things that they do not yet have or that belong to others (often called the 'house money' effect). Researchers have tested for this effect mostly in the context of public goods (e.g., Clark, 2002; Cherry et al., 2005; Epley et al., 2006; Jing and Cheo, 2013). In the context of discounting, Morrison and Oxoby (2013) found that personal discount rates were higher for endowments compared to 'found money'.

\subsection{Estimation}

We model the discount rate using grouped maximum likelihood estimation (grouped MLE) with the INTREG procedure in STATA. Cameron and Huppert (1989) discuss the grouped MLE method in the context of nonmarket valuation surveys. An alternative approach is to estimate a binary choice model of each tradeoff question, rather than using just the bounds of each respondent's discount rate range. This would increase the number of observations five-fold, but would not make use of any additional information from the respondents.

Most of our respondents always chose SS (54.4 per cent) or LL (21.4 per cent) throughout the sequence, revealing the upper or lower bound on their discount rate, but not both (i.e., single-bounded discount rate ranges). Missing upper bounds (i.e., respondents who always chose SS) are treated as censored. Missing lower bounds (i.e., respondents who always choosing LL) were replaced with 0 . Based on variation in respondent and household characteristics, we obtain an estimate of the personal discount rate $\hat{\rho}$ of all survey respondents, which is used to model environmental health behaviors.

The basic estimating equation for the discount rate is:

$$
\left(\rho_{\text {Low }, j}, \rho_{\text {High }, j}\right)=\beta_{\rho} \cdot Z_{\rho, j}+\varepsilon_{j},
$$

where $\rho_{\text {Low }}$ and $\rho_{\text {High }}$ are implied discount rates that comprise the lower and upper bound of each respondent's discount rate, and $\beta_{\rho}$ are the coefficients for characteristics $Z_{\rho}$. The estimated discount rate $\hat{\rho}_{j}$ is calculated as $\hat{\rho}=\hat{\beta}_{\rho} \cdot Z_{\rho, j}$, where $\hat{\beta}_{\rho}$ is the estimated coefficient for $Z_{\rho}$. We assume a standard normal distribution with a minimum discount rate of zero. Botelho et al. (2006); Olson and Bailey (1981) and Poulos and Whittington (2000) provide support for non-negative discounting in developing countries. We maximize the following log likelihood function:

$$
L=\prod_{j}\left[\phi\left(\frac{u_{j}-\beta_{\rho} Z_{\rho, j}}{\sigma}\right)-\phi\left(\frac{l_{j}-\beta_{\rho} Z_{\rho, j}}{\sigma}\right)\right]
$$


where $j$ is an index of respondents, $u$ and $l$ are the upper and lower bounds, respectively, of the true discount rate, and $\sigma$ is the standard deviation.

Next, the estimated discount rate $(\hat{\rho})$ is included as an explanatory variable in logit regressions on the probability that the household: (i) washes hands before eating and after defecation; (ii) treats drinking water using any method; (iii) owns a personal latrine; (iv) cooks with clean fuels such as LPG; and (v) owns a bednet.

$$
\log \frac{\left(P_{j}=1\right)}{1-\left(P_{j}=1\right)}=\beta_{0}+\beta_{1} \cdot \hat{\rho}+\beta_{Y} \cdot Z_{Y, j}+\tau_{j}
$$

where $P_{j}=1$ is the probability that the respondent chooses to adopt the environmental health behavior, and $\beta_{1}$ and $\beta_{Y}$ are coefficients for personal discount rates $(\hat{\rho})$ and other determinants of behavior $Z_{Y}$, respectively. Since the discount rate is estimated rather than observed, we estimate the standard errors of the regression coefficients in the health behavior models by bootstrapping the data set 500 times (Mooney and Duval, 1993). That is, we build 500 data sets by randomly sampling observations in the original data set with replacement. Both stages (discount rate and health behavior) of the model are estimated for the original and bootstrapped data sets. We report the point estimates for each coefficient $\left(\beta_{0}, \beta_{1}\right.$ and $\left.\beta_{Y}\right)$ from the original (non-bootstrapped) data set, and use the 500 point estimates from the bootstrapped data sets to calculate standard errors. In table 2, we show the point estimate and the $z$-statistic (which is simply the ratio of the coefficient estimate and its standard error). Stars indicate statistical significance based on the critical values for the $z$-statistics.

In the online appendix, we re-estimate this model using a measure of time preferences rather than personal discount rates. Time preferences are estimated by modeling responses to the time tradeoff questions as a function of age and gender, including numeracy and district fixed effects to control for factors that may have influenced implementation or understanding of the questions. In online appendix table A4, we report estimation results for models of environmental health behaviors as functions of the estimated rate of time preference, with covariates including credit access and socioeconomic status (see table A4). This contrasts with the models presented in table 2, which include credit access and socio-economic status in both stages, allowing those variables to affect environmental health behaviors both directly and through the personal discount rate. The supplemental models in the online appendix serve as a test of whether responses to the time tradeoff questions include information about time preferences distinct from socio-economic status and credit constraints.

\section{Results}

\subsection{Estimating personal discount rates}

In table 1, we present the results of the first stage of our empirical approach - estimating personal discount rates as a function of exogenous factors $Z_{\rho}$ (equation (3)). Based on this model, the predicted monthly discount rate in our sample varies from 9.3 to 28.7 per cent, with a mean of 16.7 
Table 1. Regression model of discount rates ${ }^{a}$

\begin{tabular}{lc}
\hline Variable & Discount rate $(\rho)^{b}$ \\
\hline Female $(\mathrm{Y}=1 ; \mathrm{N}=0)$ & $-0.074(2.28)^{*}$ \\
Age (years) & $-0.001(1.33)$ \\
Female $\times$ Age & $0.001(1.50)$ \\
Can do household accounts $(\mathrm{Y}=1 ; \mathrm{N}=0)$ & $-0.001(0.12)$ \\
Can access credit $(\mathrm{Y}=1 ; \mathrm{N}=0)$ & $-0.019(2.87)^{* *}$ \\
Household size (members) & $0.002(1.51)$ \\
Expenditure on staple foods $(\%)$ & $0.001(2.70)^{* *}$ \\
Wealth index (principal component) & $-0.005(1.49)$ \\
Observations $(n)^{c}$ & 8,040 \\
\hline Estimated discount rates & \\
\hline Mean (monthly) & $16.7 \%$ \\
Range (monthly) & $9.3-28.7 \%$ \\
\hline
\end{tabular}

Notes: ${ }^{a}$ Controlling for district effects, not reported in the table. ${ }^{b}$ Coefficients; $t$-statistics in brackets; statistical significance: ${ }^{*} p<0.1 ;{ }^{* *} p<0.05$. ${ }^{c}$ Excludes households with inconsistent or missing responses to time tradeoff questions $(1,908)$, and/or missing values of other variables (316).

per cent per month. The distribution is bi-modal, with two peaks around 13 and 19 per cent. Although the estimated discount rates may appear too high in comparison to those in the developed countries, they are commensurate with the distribution of interest rates reported by respondents who can access formal credit. Of the respondents who could access any type of formal credit, half of them reported that they could obtain a loan with interest equal to or higher than 5 per cent per month. Respondents who believe that they cannot access formal credit (and therefore did not answer this question) would arguably have to pay even higher effective rates for informal credit (Barrett, 2007).

The relationships between discount rates revealed by the time tradeoff questions and sex, economic status (i.e., percent expenditures on staple food, household size and principal component of assets) and access to formal credit are consistent with the prior literature (see online appendix table A1). However, we do not find the expected U-shaped relationship with age. Square and cubic terms are omitted from the final specification because they were found to be statistically insignificant. We do find a weak correlation between responses to the time tradeoff questions and an interaction term of age and sex. The coefficients on age, sex and the interaction term are jointly significant (Prob $>\chi^{2}(3)=0.05$ ). The ability to do household accounts, which proxies for numeracy, is not statistically significant in the model. The model also includes fixed effects for districts.

\subsection{The link between discount rates and disease prevention}

In table 2, we present the results of the second stage of our empirical approach - the relationships between estimated personal discount rates and the households' options for reducing environmental health burdens. 
Table 2. Environmental health behaviors and estimated personal discount rates $(n=9,750)$

\begin{tabular}{|c|c|c|c|c|c|}
\hline Variable & $B N E T^{a}$ & $I H L^{a}$ & CFUEL ${ }^{a}$ & TWATER $^{a}$ & $H W^{a}$ \\
\hline Estimated discount rate & $-10.455(8.19)^{* * *}$ & $-9.358(5.78)^{* * *}$ & $-14.814(7.10)^{* * *}$ & $-2.309(1.45)^{\S}$ & $-5.749(4.10)^{* * *}$ \\
\hline $\begin{array}{l}\text { Can access credit } \\
\quad(\mathrm{Y}=1 ; \mathrm{N}=0)\end{array}$ & $0.121(1.41)$ & $0.088(0.88)$ & $-0.011(0.08)$ & $0.106(1.88)^{*}$ & $-0.225(3.48)^{* * *}$ \\
\hline Household size (members) & $-0.001(0.03)$ & $0.014(0.79)$ & $-0.051(1.98)^{* *}$ & $-0.019(1.89)^{*}$ & $0.012(1.06)$ \\
\hline $\begin{array}{l}\text { Expenditure on staple foods } \\
(\%)\end{array}$ & $-0.013(2.66)^{* * *}$ & $-0.02(2.55)^{* *}$ & $-0.05(3.32)^{* * *}$ & $0.013(5.80)^{* * *}$ & $-0.005(1.99)^{* *}$ \\
\hline $\begin{array}{l}\text { Wealth index (principal } \\
\text { component) }\end{array}$ & $0.298(6.11)^{* * *}$ & $0.679(13.33)^{* * *}$ & $0.331(5.09)^{* * *}$ & $0.032(1.25)$ & $0.092(3.01)^{* * *}$ \\
\hline $\begin{array}{l}\text { Total monthly } \\
\text { expenditure (Rs.) }\end{array}$ & $1.347(2.27)^{* *}$ & $2.501(3.88)^{* * *}$ & $4.128(5.12)^{* * *}$ & $2.243(3.01)^{* * *}$ & $-0.432(0.76)$ \\
\hline $\begin{array}{l}\text { Concerned about diseases in } \\
\text { village? }(\mathrm{Y}=1 ; \mathrm{N}=0)\end{array}$ & $-0.161(2.52)^{* *}$ & $0.017(0.19)$ & $-0.377(4.59)^{* * *}$ & $0.162(3.14)^{* * *}$ & $0.109(2.06)^{* *}$ \\
\hline $\begin{array}{l}\text { Faced crisis in last } 12 \text { months } \\
\qquad(\mathrm{Y}=1 ; \mathrm{N}=0)\end{array}$ & $0.021(0.29)$ & $-0.124(1.25)$ & $-0.306(2.84)^{* * *}$ & $-0.008(0.12)$ & $0.113(1.91)^{*}$ \\
\hline Distance to clinic (min) & $-0.005(5.40)^{* * *}$ & $-0.009(5.67)^{* * *}$ & $-0.01(5.36)^{* * *}$ & $-0.002(3.15)^{* * *}$ & $-0.001(1.65)^{*}$ \\
\hline $\begin{array}{l}\text { Distance to village } \\
\text { center (min) }\end{array}$ & $-0.005(1.88)^{*}$ & $-0.018(3.81)^{* * *}$ & $-0.011(2.92)^{* * *}$ & $0.006(3.81)^{* * *}$ & $-0.003(2.77)^{* * *}$ \\
\hline Formal education (years) & $0.212(6.81)^{* * *}$ & $0.264(7.53)^{* * *}$ & $0.233(6.20)^{* * *}$ & $0.046(1.80)^{*}$ & $0.042(1.75)^{*}$ \\
\hline Can read? $(Y=1 ; N=0)$ & $0.31(3.43)^{* * *}$ & $0.577(5.28)^{* * *}$ & $0.828(6.30)^{* * *}$ & $0.051(0.84)$ & $0.275(4.25)^{* * *}$ \\
\hline Constant & $-0.106(0.42)$ & $-1.597(5.04)^{* * *}$ & $0.034(0.08)$ & $0.508(1.72)^{*}$ & $0.508(1.88)^{*}$ \\
\hline
\end{tabular}

Notes: ${ }^{a}$ Coefficients; $z$-statistics in parentheses; standard errors computed through bootstrapping. Statistical significance: ${ }^{8} p<0.15$; ${ }^{*} p<0.1{ }^{* *} p<0.05 ;{ }^{* * *} p<0.01$. 
All environmental health behaviors are negatively correlated with the estimated discount rate, even after controlling for a suite of covariates. The estimated coefficients on the covariates generally have the expected signs. For example, literacy and years of formal education are positively related to the probability of engaging in all of these behaviors. Current disposable income (proxied by total household expenditure) is positively related to all behaviors, although the relationship is weak with handwashing, possibly because it requires the least cash outlay.

In the online appendix, we report estimation results using the rate of time preference, derived from modeling the responses to the time tradeoff questions as a function only of intrinsic personal characteristics, i.e., age and sex, and factors that may have influenced implementation of the survey, i.e., numeracy and district fixed effects, specifically excluding variables representing socio-economic status and access to credit. We do include socio-economic status and credit access in the second stage alongside this estimated rate of time preference. Qualitatively there are practically no differences from table 2 - most environmental health behaviors are also negatively correlated with time preferences. This provides some evidence that responses to the time tradeoff questions include information about how the respondents make temporal tradeoffs that are distinct from socio-economic status and credit constraints.

\section{Discussion}

Our study adds to a small but growing body of empirical literature from developing countries on the determinants of personal discount rates and their relationships with health behaviors. We find that discount rates are negatively related with a wide range of actions that households can take to prevent health problems, including point-of-use water treatment, handwashing, cooking with clean fuel, and ownership of household latrines and bednets (table 2). This correlation between lower discount rates and greater household investment in behaviors that avert environmental health problems confirms the importance of testing policy alternatives such as credit and payment schemes (e.g., Pattanayak et al., 2006; Guiteras et al., 2016). However, we find that discount rates are inversely related even with handwashing, which requires effort to establish the habit but little time or cash outlay. This finding, together with the statistical significance of time preferences in models of environmental health behaviors (see table A4), suggests that these relationships may reflect intrinsic preferences that will be difficult to influence with external interventions and policies.

Using the estimated coefficients and characteristics of the average respondent to calculate marginal effects, we find that a 10 percentage point decrease in the monthly discount rate (which is half a standard deviation decrease) is associated with increases in the probability of treating water by 2 per cent, washing hands by 6 per cent, having a latrine by 14 per cent, cooking with clean fuels by 23 per cent, and owning a bednet by 14 per cent. Although we do not interpret these as causal effects, it is worth noting that they are quite large relative to the frequency of these behaviors (table 2), as might be expected from a large (10 per cent) change in the discount rate. 
The estimated discount rates are higher than those typically used in costbenefit analysis in developing countries. For example, the Asian Development Bank follows convention among international development agencies in using an annual discount rate of 10-12 per cent for economic analyses of their development projects (ADB Economic and Development Resource Center, 1997). Kula (2004) estimates the Indian social discount rate at 5.2 per cent per year. However, personal discount rates are typically higher than social discount rates (Jha, 1998). The discount rates revealed in our survey may also reflect the fact that most respondents have limited access to formal credit and therefore face very high effective interest rates for informal forms of credit. One caveat is that the discount rates revealed through the time tradeoff questions may be influenced by the 'found money' effect (Morrison and Oxoby, 2013). In general, we suggest that discount rates elicited through survey methods are best viewed as coarse indicators that can help explain variation in behaviors across households, rather than precise estimates to perform net present value calculations.

To reduce concerns about model misspecification due to omitted variables, our analyses include key covariates such as concern about disease in the village, distances to nearest clinic and the village center, current economic conditions (measured by monthly expenditures and recent household crises), and proxies for household socio-economic status, including education and literacy as well as wealth, percentage of expenditures on staple food, and household size. Because our focus is on discount rates, we treat these covariates as controls and do not discuss their estimated coefficients in detail. In brief, many of these covariates have statistically significant effects, confirming that both human capital (knowledge and ability to access information) and income are important factors in the adoption of these environmental health behaviors (Pattanayak and Pfaff, 2009).

In summary, we find that personal discount rates are lower for women, for better-off households, and for households that can access formal credit. In turn, lower discount rates are correlated with increased probabilities of adopting behaviors that reduce future harm from environmental risks, such as handwashing, water treatment, and the use of bednets, clean cooking fuels and individual household latrines. To our knowledge, this is the first empirical test of the relationship between personal discount rates and household adoption of environmental health technologies among the rural poor in developing countries. This is important because these behavioral choices are critical for health and development outcomes and, ultimately, escaping poverty (Pattanayak and Pfaff, 2009; Dasgupta, 2013).

\section{Supplementary material and methods}

To view supplementary material for this article, please visit https://doi. org/10.1017/S1355770X17000018.

\section{References}

ADB Economic and Development Resource Center (1997), Guidelines for the Economic Analysis of Projects, Manila: Asian Development Bank. 
Agee, M. and T. Crocker (2000), 'Household environmental protection and the intergenerational transmission of human capital', Journal of Economic Psychology 21(6): 673-690.

Anderson, C.L. and M.K. Gugerty (2009), 'Intertemporal choice and development policy: new evidence on time-varying discount rates from Vietnam and Russia', Developing Economies 47(2): 123-146.

Anderson, C.L., M. Dietz, A. Gordon, and M. Klawitter (2004), 'Discount rates in Vietnam', Economic Development and Cultural Change 52(4): 873-887.

Arya, S., C. Eckel, and C. Wichman (2013), 'Anatomy of the credit score', Journal of Economic Behavior and Organization 95(November): 175-185.

Ashraf, N., D. Karlan, and W. Yin (2006), 'Tying Odysseus to the mast: evidence from a commitment savings product in the Philippines', Quarterly Journal of Economics 121(2): 635-672.

Barrett, C.B. (2007), 'Displaced distortions: financial market failures and seemingly inefficient resource allocation in low-income rural communities', in E. Bulte and R. Ruben (eds), Development Economics Between Markets and Institutions: Incentives for Growth, Food Security and Sustainable Use of the Environment, Wageningen: Wageningen Academic Publishers, pp. 73-86.

Bauer, M. and J. Chytilová (2009), ‘Do children make women more patient? Experimental evidence from Indian villages', IES Working Paper No. 10/2009, Charles University, Faculty of Social Sciences, Institute of Economic Studies, Prague.

Bauer, M. and J. Chytilová (2010), 'The impact of education on subjective discount rate in Ugandan villages', Economic Development and Cultural Change 58(4): 643-669.

Bauer, M., J. Chytilová, and J. Morduch (2012), 'Behavioral foundations of microcredit: experimental and survey evidence from rural India', American Economic Review 102(2): 1118-1139.

Becker, G.S. and C.B. Mulligan (1997), 'The endogenous determination of time preference', Quarterly Journal of Economics 112(3): 729-758.

Botelho, A., G.W. Harrison, L.M.C. Pinto, E.E. Rutström, and P. Veiga (2006), 'Discounting in developing countries: experimental evidence from Timor-Leste', Faculty Working Paper No. 31, Economics Department, University of Central Florida, Orlando, FL.

Bradford, W.D. (2010), 'The association between individual time preferences and health maintenance habits', Medical Decision Making 30(1): 99-112.

Bruce, N., E. Rehfuess, S. Mehta, G. Hutton, and K. Smith (2006), 'Indoor air pollution', in D.T. Jamison, J.G. Breman, A.R. Measham, et al. (eds), Disease Control Priorities in Developing Countries II, New York: Oxford University Press, pp. 793-816.

Cairncross, S. and V. Valdmani (2006), 'Water supply, sanitation, and hygiene promotion', in D.T. Jamison, J.G. Breman, A.R. Measham, et al. (eds), Disease Control Priorities in Developing Countries II, New York: Oxford University Press, pp. 771-792.

Cameron, T.A. and D. Huppert (1989), 'OLS versus ML estimation of non-market resource values with payment card interval data', Journal of Environmental Economics and Management 17(3): 230-246.

Cardenas, J.C. and J.P. Carpenter (2008), 'Behavioural development economics: lessons from field labs in the developing world', Journal of Development Studies 44(3): 311-338.

Carpenter, J., G. Harrison, J. List, and B.A.P. Taxonomy (2005), 'Field experiments in economics: an introduction', Field Experiments in Economics 10(1): $1-15$. 
Chabris, C.F., D. Laibson, C.L. Morris, J.P. Schuldt, and D. Taubinsky (2008), 'Individual laboratory-measured discount rates predict field behavior', Journal of Risk and Uncertainty 37(2-3): 237-267.

Chapman, G.B. (2005), 'Short-term cost for long-term benefit: time preference and cancer control', Health Psychology 24(4): S41-S48.

Cherry, T.L., S. Kroll, and J.F. Shogren (2005), 'The impact of endowment heterogeneity and origin on public good contributions: evidence from the lab', Journal of Economic Behavior \& Organization 57(3): 357-365.

Chesson, H.W., J.S. Leichliter, G.D. Zimet, S.L. Rosenthal, D.I. Bernstein, and K.H. Fife (2006), 'Discount rates and risky sexual behaviors among teenagers and young adults', Journal of Risk and Uncertainty 32(3): 217-230.

Clark, J. (2002), 'House money effects in public good experiments', Experimental Economics 5(3): 223-231.

Dasgupta, P. (2013), 'Personal histories and poverty traps', in C. Sepulveda, A. Harrison and J.Y. Lin (eds), Development Challenges in a Post-crisis World: Annual Bank Conference on Development Economics 2011, Washington, DC: World Bank, pp. 103-126.

Dixon, M.R., J. Marley, and E.A. Jacobs (2003), 'Delay discounting by pathological gamblers', Journal of Applied Behavior Analysis 36(4): 449-458.

Eckel, C., C. Montmarquette, and C. Johnson (2004), 'Saving decisions of the working poor: short- and long-term horizons', CIRANO Working Paper 2004s-45, Montreal.

Ekholm, T., V. Krey, S. Pachauri, and K. Riahi (2010), 'Determinants of household energy consumption in India', Energy Policy 38(10): 5696-5707.

Epley, N., D. Mak, and L.C. Idson (2006), 'Bonus of rebate? The impact of income framing on spending and saving', Journal of Behavioral Decision Making 19(3): 213-227.

Fewtrell, L., R.B. Kaufmann, D. Kay, W. Enanoria, L. Haller, and J.M. Colford, Jr. (2005), 'Water, sanitation, and hygiene interventions to reduce diarrhoea in less developed countries: a systematic review and meta-analysis', Lancet Infectious Diseases 5(1): 42-52.

Filmer, D. and L.H. Pritchett (2001), 'Estimating wealth effects without expenditure data - or tears: an application to educational enrollments in states of India', Demography 38(1): 115-132.

Fong, G.T. and P.A. Hall (2003), 'The importance of time perspective in predicting, understanding, and reducing health risk behaviors among adolescents', in D. Romer (ed.), Reducing Adolescent Risk: Toward an Integrated Approach, Newbury Park, CA: Sage, pp. 106-112.

Frederick, S., G. Loewenstein, and T. O'Donoghue (2002), 'Time discounting and time preference: a critical review', Journal of Economic Literature 40(2): 351-401.

Godoy, R., M. Jacobson, J. de Castro, V. Aliaga, J. Romero, and A. Davis (1998), 'The role of tenure security and private time preference in neotropical deforestation', Land Economics 74(2): 162-170.

Guiteras, R.P., D.I. Levine, T.H. Polley, and B. Quistorff (2016), 'Credit constraints, discounting and investment in health: evidence from micropayments for clean water in Dhaka', [Available at] http:/ / www.economics.cornell.edu/sites/default/ files/files/events/GLPQ\%20-\%20Dhaka\%20Water\%20WTP\%20-\%20Feb\%202014. Dev_.Aug_.28pdf.pdf.

Hall, P.A.H. and G.T. Fong (2007), 'Temporal self-regulation theory: a model for individual health behavior', Health Psychology Review 1(1): 6-52.

Harrison, G.W., M.I. Lau, and M.B. Williams (2002), 'Estimating individual discount rates in Denmark: a field experiment', American Economic Review 92(5): 1606-1617. 
Henrich, J., S.J. Heine, and A. Norenzayan (2010), 'The weirdest people in the world?', Behavioral and Brain Sciences 33(2-3): 61-83.

Holden, S.T., B. Shiferaw, and M. Wik (1998), 'Poverty, market imperfections and time preferences: of relevance for environmental policy?', Environment and Development Economics 3(1): 105-130.

Jalan, J., E. Somanathan, and S. Chaudhuri (2009), 'Awareness and the demand for environmental quality: drinking water in urban India', Discussion Papers in Economics No. 03-05, Indian Statistical Institute, Planning Unit, New Delhi.

Jha, R. (1998), Modern Public Economics, London: Routledge.

Jing, L. and R. Cheo (2013), 'House money effects, risk preferences and the public goods game', Economics Letters 120(2): 310-313.

Johnson, M.W. and W.K. Bickel (2002), 'Within-subject comparison of real and hypothetical money rewards in delay discounting', Journal of the Experimental Analysis of Behavior 77(2): 129-146.

Khwaja, A., F. Sloan, and M. Salm (2006), 'Evidence on preferences and subjective beliefs of risk takers: the case of smokers', International Journal of Industrial Organization 24(4): 667-682.

Kirby, K.N. and N.M. Petry (2004), 'Heroin and cocaine abusers have higher discount rates for delayed rewards than alcoholics or non-drug-using controls', Addiction 99(4): 461-471.

Kirby, K., R. Godoy, V. Reyes-Garcia, et al. (2002), 'Correlates of delay-discount rates: evidence from Tsimane' Amerindians of the Bolivian rain forest', Journal of Economic Psychology 23: 291-316.

Kula, E. (2004), 'Estimation of a social rate of interest for India', Journal of Agricultural Economics 55(1): 91-99.

Lawless, L., A.C. Drichoutis, and R.M. Nayga, Jr. (2013), 'Time preferences and health behaviour: a review', Agricultural and Food Economics 1(1): 1-19.

Levy, A.G., E. Micco, M. Putt, and K. Armstrong (2006), 'Value for the future and breast cancer-preventive health behavior', Cancer Epidemiology Biomarkers $\mathcal{E}$ Prevention 15(5): 955-960.

Lewis, J.J. and S.K. Pattanayak (2012), 'Who adopts improved fuels and cookstoves? A systematic review', Environmental Health Perspectives 120(5): 637-645.

Liebenehm, S. and H. Waibel (2014), 'Simultaneous estimation of risk and time preferences among small-scale cattle farmers in West Africa', American Journal of Agricultural Economics 96(5): 1420-1438.

List, J.A., and C.A. Gallet (2001), 'What experimental protocol influence disparities between actual and hypothetical stated values? Evidence from a meta-analysis', Environmental and Resource Economics 20(3): 241-254.

Luby, S.P., C. Mendoza, B.H. Keswick, T.M. Chiller, and R.M. Hoekstra (2008), 'Difficulties in bringing point-of-use water treatment to scale in rural Guatemala', American Journal of Tropical Medicine and Hygiene 107(2): 573-597.

Luby, S.P., M. Agboatwalla, A. Bowen, E. Kenah, Y. Sharker, and R.M. Hoekstra (2009), 'Difficulties in maintaining improved handwashing behavior, Karachi, Pakistan', American Journal of Tropical Medicine and Hygiene 81(1): 140-145.

Lumley, S. (1997), 'The environment and the ethics of discounting: an empirical analysis', Ecological Economics 20(1): 71-82.

Meerding, W.J., G.J. Bonsel, W.B.F. Brouwer, M.C. Stuifbergen, and M. EssinkBot (2010), 'Social time preferences for health and money elicited with a choice experiment', Value in Health 13(4): 368-374.

Meier, S. and C. Sprenger (2010), 'Present-biased preferences and credit card borrowing', American Economic Journal: Applied Economics 2(1): 193-210.

Mooney, C.Z. and R.D. Duval (1993), Bootstrapping: A Nonparametric Approach to Statistical Inferences, Newbury Park, CA: Sage. 
Moore, M.J. and W.K. Viscusi (1988), 'The quantity-adjusted value of life', Economic Inquiry 26(3): 369-388.

Morrison, W.G. and R.J. Oxoby (2013), 'The endowment effect and intertemporal choice: a laboratory investigation', Canadian Journal of Economics/Revue Canadienne d'Économique 46(2): 689-704.

Murphy, J.J., P.G. Allen, T.H. Stevens, and D. Weatherhead (2003), 'A meta-analysis of hypothetical bias in stated preference valuation', Working Paper No. 2003-8, Department of Resource Economics, University of Massachusetts, Amherst, MA.

Nagin, D.S. and G. Pogarsky (2004), 'Time and punishment: delayed consequences and criminal behavior', Journal of Quantitative Criminology 20(4): 295-317.

Nguyen, Q., M.C. Villeval, and H. Xu (2016), 'Trust under the prospect theory and quasi-hyperbolic preferences: a field experiment in Vietnam', Economic Development and Cultural Change 64(3): 545-572.

Nielsen, U. (2001), 'Poverty and attitudes towards time and risk: experimental evidence from Madagascar', Working Paper, Department of Economics and Natural Resources, Royal Veterinary and Agricultural University, Frederiksberg, Denmark.

Olson, M. and M.J. Bailey (1981), 'Positive time preference', Journal of Political Economy 89(1): 1-25.

Orbell, S., M. Perugini, and T. Rakow (2004), 'Individual differences in sensitivity to health communications: consideration of future consequences', Health Psychology Review 23(4): 388-396.

Pant K., S.K. Pattanayak, and M. Thakuri (2014), 'Climate change, cook stoves, and coughs and colds: thinking global and acting local in rural Nepal', in S. Barrett, K.-G. Mäler and E. Maskin (eds), Environment and Development Economics: Essays in Honour of Sir Partha Dasgupta, Oxford: Oxford University Press, pp. 145-168.

Pattanayak, S.K. and A. Pfaff (2009), 'Behavior, environment, and health in developing countries: evaluation and valuation', Annual Review of Resource Economics 1(1): 183-217.

Pattanayak, S.K., C. Van den Berg, J.-C. Yang, and G. Van Houtven (2006), 'The use of willingness to pay experiments: estimating demand for piped water connections in Sri Lanka', World Bank Policy Research Working Paper No. 3818, Washington, DC.

Pattanayak, S.K., C. Poulos, J.-C. Yang, S.R. Patil, and K.J. Wendland (2009), 'Of taps and toilets: quasi-experimental protocol for evaluating community-demanddriven projects', Journal of Water and Health 7(3): 434-451.

Pattanayak, S.K., J.-C. Yang, C. Poulos, and S.R. Patil (2010), 'How valuable are environmental health interventions? Evaluation of water and sanitation programs in India', Bulletin of the World Health Organization 88(7): 535-542.

Pender, J.L. (1996), 'Discount rates and credit markets: theory and evidence from rural India', Journal of Development Economics 50(2): 257-296.

Picone, G., F.A. Sloan, and D.J. Taylor (2004), 'Effects of risk and time preference and expected longevity on demand for medical tests', Journal of Risk and Uncertainty 28(1): 39-53.

Poulos, C. and D. Whittington (2000), 'Time preferences for life-saving programs: evidence from six less developed countries', Environmental Science and Technology 34(8): 1445-1455.

Rieger, M. (2015), 'Risk aversion, time preference and health production: theory and empirical evidence from Cambodia', Economics \& Human Biology 17(1):1-15.

Rogers, A.R. (1994), 'Evolution of time preference by natural selection', American Economic Review 84(3): 460-481. 
Rubalcava, L., G. Teruel, and D. Thomas (2009), 'Investments, time preferences and public transfers paid to women', Economic Development and Cultural Change 57(3): 507-538.

Samuelson, P. (1937), 'A note on the measurement of utility', Review of Economic Studies 4(2): 151-161.

Scharff, R.L. and W.K. Viscusi (2011), 'Heterogeneous rates of time preference and the decision to smoke', Economic Inquiry 49(4): 959-972.

Shanmugam, K.R. (2006), 'Rate of time preference and the quantity adjusted value of life in India', Environment and Development Economics 11(5): 569-583.

Story, G.W., I. Vlaev, B. Seymour, A. Darzi, and R.J. Dolan (2014), 'Does temporal discounting explain unhealthy behavior? A systematic review and reinforcement learning perspective', Frontiers in Behavioral Neuroscience 8(1): 76.

Sutter, M., M.G. Kocher, and S.T. Trautmann (2013), 'Impatience and uncertainty: experimental decisions predict adolescents' field behavior', American Economic Review 103(1): 510-531.

Tanaka, T., C.C. Camerer, and Q. Nguyen (2010), 'Risk and time preferences: linking experimental and household survey data from Vietnam', American Economic Review 100(1): 557-571.

Tsukayama, E. and A.L. Duckworth (2010), 'Domain-specific temporal discounting and temptation', Judgment and Decision Making 5(2): 72-82.

UNICEF (2009), 'Diarrhoea: why children are still dying and what can be done', [Available at] http:/ / www.who.int/child_adolescent_health/documents / 9789241598415/en/index.html.

Vuchinich, R.E. and C.A. Simpson (1998), 'Hyperbolic temporal discounting in social drinkers and problem drinkers', Experimental and Clinical Psychopharmacology 6(3): 292-305.

WCED (World Commission on Environment and Development) (1987), Our Common Futures, Oxford: Oxford University Press.

Wendland, K.J., S.K. Pattanayak, and E.O. Sills (2014), ‘Do national-level differences matter in the adoption of environmental health technologies? A cross-border comparison from Benin and Togo', Health Policy and Planning 30(2): 145-154.

World Bank (2008), Of Taps \& Toilets: Evaluating Community Demand-Driven Projects in Rural India. Environment, Water Resource and Climate Change, Washington, DC: South Asia Division, World Bank.

Yesuf, M. (2004), 'Risk, time and land management under market imperfections: applications to Ethiopia', PhD dissertation, Göteborg University, Sweden. 